\title{
A driver-distraction-based lane-keeping assistance system
}

J Pohl*, W Birk, and L Westervall

Vehicle Control and Active Safety, Volvo Car Corporation, Gothenburg, Sweden

The manuscript was received on 10 November 2005 and was accepted after revision for publication on 29 November 2006.

DOI: 10.1243/09596518JSCE218

\begin{abstract}
Single-vehicle roadway departure (SVRD) accidents occur in many cases owing to driver distraction or drowsiness constituting a substantial share of today's road vehicle accidents and casualties. This paper describes a distraction-based lane-keeping support system, which intervenes only when the driver is positively detected as being distracted. Distraction here is understood as cognitive and visual distraction, and the focus of this system is on the latter one. In order to estimate the driver's visual distraction level, a video-based driver monitoring system is used. Lane-keeping support is provided by an additional torque applied on the steering shaft in order to regain an appropriate lane position. In this manner the system only intervenes when the vehicle has drifted out of its lane and while the driver is distracted. Test track investigations indicate large opportunities for such a system from a driver perspective, provided that sufficient reliability of the employed vision sensor for lane and face tracking can be obtained.
\end{abstract}

Keywords: visual distraction, lane keeping, eye tracking, roadway departure accident

\section{INTRODUCTION}

Many single-vehicle roadway departure (SVRD) accidents take place in uncritical traffic situations and good weather conditions. According to accident data over the last 10 years from German roadways [1], 14.5 per cent of all accidents were due to unintended lane departure, 35 per cent of these with deadly consequences. Reasons for lane departure are many and range from the driver's inability to control the vehicle (for example, on account of severe weather conditions) to lack of attention, drowsiness, and intoxication or illness leading to the loss of lanekeeping control. A study by the National Highway Traffic Safety Administration (NHTSA) [2] indicates that 25 per cent of all drivers use cell phones for either inbound or outbound calls, and 24 per cent of all drivers deal with children in the back seat. Additionally, among the drivers that have been involved in a crash within the last 5 years, 3.5 per

\footnotetext{
* Corresponding author: Vehicle Control and Active Safety, Volvo Car Corporation, PVH 34, Volvo Jacobs Väg, Gothenburg 405 31, Sweden.email: jpohl12@volvocars.com
}

cent related the accident cause to being distracted. Additionally, in reference [3] it is summarized how in-vehicle telematics are affecting a driver's workload and the resulting safety implications. This survey also indicates the value of support systems for the driver in order to reduce the risk of accidents.

Today, a common approach to reducing lane departure is the use of so-called 'rumble strips'. Rumble strips are areas of grooved pavement or grooved lane marking that can either be placed approximately $15 \mathrm{~cm}$ beyond the lane boundary or be implemented in the lane marking itself. When the vehicle leaves the lane, the tyres contact the rumble strip and thereby produce a noise that alerts the driver. However, rumble strips have yet to spread to all countries, and the shaping in terms of produced noise differs.

An alternative approach, which does not require infrastructure modification, is to use a system that can detect when the driver is beginning to depart from the road, and thereby sound an alarm or activate a haptic feedback in time for the driver to take corrective action. These types of system are known as lane departure warning/lane-keeping systems and use sensors (most often cameras with 
Table 1 Summary of warning/intervention modalities in terms of their performance

\begin{tabular}{lll}
\hline Sound warning has been taken as datum & Haptic warning & Haptic intervention \\
False warning/intervention acceptance & 0 & -- \\
True warning effectiveness & 0 & ++ \\
Driver acceptance & + & + \\
Association between warning/intervention and action & Depends on modality & + \\
\hline
\end{tabular}

real-time image processing) to track features on the road such as lane markings. From this information the vehicle lane position is determined, which can be used to warn (similarly to rumble strips) or actually to predict when the driver is at risk of departing from the road and warn accordingly. Predictive warning is the main advantage over rumble strips, besides the fact that no infrastructure modifications are needed. However, predictive warning might result in driver annoyance due to a higher warning frequency, even during normal driving.

Lane-keeping assistance systems have recently been introduced on the Japanese market. These systems aid the driver in lateral vehicle control by providing an additional torque on the steering shaft to guide the driver into the right direction. A disadvantage of these systems becomes apparent when a lane change or lane departure manoeuvre is considered. These systems usually cannot distinguish between intentional and unintentional lane departures. Such an indication is often done by using the directional indicator, thus signalling that an intentional lane change can be expected.

The advantage with this type of lane-keeping system is that the haptic warning through the steering wheel, compared with a sound, is provided to the driver only, and does not disturb other passengers in the vehicles. A sound warning can be difficult to associate with a required driver action, especially if the intervention is rare. However, this is not the case for lane departure warning systems. On the other hand, haptic warnings through the steering wheel can be annoying if, for instance, the driver performs a lane change without using the indicator, which will trigger a warning.

However, from an ergonomic point of view, haptic and sound warnings have similar performance in terms of driver reaction. Even the amount of false warnings or interventions, that is, interventions that occur without obvious reason for the driver, must be taken into account when choosing the driver interface. Table 1 summarizes the different warning/ intervention modalities in terms of their performance.

However, there is a general problem with these kinds of lane-keeping/lane departure warning devices, which is the difference in perception of 'danger' by system and driver. The system wants the driver to avoid drifting out of the lane in order to prevent an accident, and therefore warns or intervenes. The driver, however, does not necessarily associate a lane marking crossing with immediate danger, as, for instance, during curve cutting manoeuvres or lane changing without indicator usage. This increases the risk of driver annoyance through such a warning or intervention. This risk is particularly apparent for the haptic intervention through the steering wheel but even exists for a warning sound 'worth its name'.

In order to cope with this shortcoming of the haptic warning, a lane-keeping assistance system has been connected to a driver distraction monitor. Thereby, the haptic warning during drifting out of lane is only actuated when the driver appears to be distracted. For the following discussion it makes sense to define intervention/warning types of a system (see Table 2).

For true negative operation, which is when the system does not intervene during intentional lane changes, the distraction module is a cure to the two 'minus signs' in Table 1 for driver acceptance of false warnings, as drivers were disturbed by a torque intervention at lane changes without indicator usage.

Another benefit of the distraction module becomes apparent when looking at false positive interventions as the distraction module will reduce the number of false interventions due to, for instance, a falsely detected lane change (false positive), as the driver has to be detected (falsely) distracted (false positive) at the same time.

From a hazard analysis point of view, the intervention authority can be increased, as the number of 'false positive' interventions will be reduced, thus leading to a constant hazard and a more effective system.

Here it is regarded that the main focus is put on avoiding false positive interventions. Missed interventions (false negative) are not regarded as critical

Table 2 Definition of intervention types

\begin{tabular}{ll}
\hline Intervention type & Description \\
\hline True positive & Correct intervention \\
True negative & Correct missed intervention (no action) \\
False positive & False intervention \\
False negative & Missed intervention \\
\hline
\end{tabular}


from a hazard analysis point of view, as the system performs in the same way as a vehicle without such a system.

In this paper the focus is on driver distraction as an accident cause, namely SVRD accidents where loss of traction under curve overspeed may not have been the accident cause. It is assumed that a system that could detect driver distraction and could intervene or warn the driver may contribute to increased road safety.

In the next section the architecture of a distractionbased lane-keeping system is presented, and the major system components such as torque intervention as well as the distraction module are explained. In the last section, results of an investigation of drivers' perception of such a system are presented.

\section{DRIVER DISTRACTION}

Distraction here is defined as not watching the road scene ahead. A similar approach in terms of conditional warning/intervention has been taken in reference [4], where road speed signs are presented to the driver via an in-car system when the system has positively detected that the driver has not looked at the sign.

In this manner, the definition of driver distraction, namely the amount of road scene attention, is quite straightforward, but the assessment of the same is quite difficult. The challenge lies in the sensor used, which is a camera with image processing monitoring the driver. During the early development stages of the distraction system, the gaze vector, meaning the direction of the individual eyeball in relation to the vehicle interior, was employed in order to find out whether the driver was watching the road scene or not. This approach is the most obvious but has the major drawback that the eyeballs must be detected in order to be able to calculate the gaze vector. This is particularly difficult when it comes to drivers wearing glasses or high dynamic lighting conditions with light and dark shadows in the driver's face.

The approach taken here makes use of the driver's face vector which in principle is the direction of the driver's nose tip. Thereby, eyeball detection is surplus. The detection of the face direction is much easier and robust, but has the disadvantage that the driver can 'cheat', meaning that he/she looks, for instance, at the radio with little head motion. However, this manoeuvre is not very comfortable in the long run, and is only expected when drivers test the system.

\subsection{Some aspects of driver distraction}

Usually, two types of distraction are distinguished: visual and cognitive. Visual distraction arises when the driver's visual focus is not on the road scene but somewhere else (e.g. the car stereo). According to reference [5], drivers are more susceptible to distractions coming from the inside of the vehicle than those from the outside, which implies that there are two basic categories for the visual focus: the road scene and the interior.

In this case the driver is not able correctly to assess the road scene and will eventually depart from the road. Since the driver may have a memory of the last assessed road scene, he/she might be able to maintain an acceptable lateral position for a certain time.

This time depends largely on the current scenario, e.g. road curvature changes, velocity, etc. Additionally, it depends on the visual focus, since certain visual focus regions might give a driver no scenario assessment while others might yield an assessment with reduced quality. For example, while looking into the rear mirror, the driver might still be able to maintain a rather good lateral position on the road, although the assessment of the road scenario is rather coarse.

Consequently, the visual distraction level can be modelled as a time dependency of the visual focus. In addition, the visual distraction level is non-linear in its nature, as visual distraction increases with time (the driver looks away from the road scene), but decreases nearly instantaneously (the driver refocuses on the road scene).

This can be illustrated by the following example. A driver has visual focus on the road but wants to change the radio channel. As soon as the visual focus turns to the car stereo the distraction level increases over time. After the task is performed and the driver returns focus to the road, the distraction level reduces back to zero again. Since the driver usually assesses the road scene instantaneously, the distraction level should return to zero in the same manner. A principal plot for such behaviour is shown in Fig. 1. It is important that a distraction estimation algorithm reflects that kind of behaviour.

When it comes to cognitive distraction, the estimation becomes much more difficult. The problem is that this type of distraction cannot be defined in terms of visual focus. It might be the case that the driver has visual focus on the road but actually is not assessing the road scene at all. In other words: 'see but not see'. Often, drivers do simply stare at a point in space, which in turn results in rather small eyeball movements. This behaviour is often exhibited by 


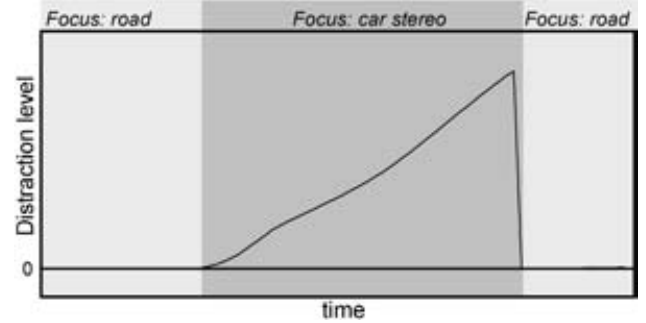

Fig. 1 Non-linear behaviour of the distraction level

drivers who are using a cell phone, and it is often also referred to as tunnel vision (see, for example, reference [6], [7], or [8]). Although this is an important type of distraction, it is extremely hard to assess its contribution to accident statistics. A cognitive distracted driver would not be detected by the system, thus leading to a false negative intervention, as the detection requires eyeball movement analysis as well as eye detection. Since it is still a matter of research to define suitable detection techniques for this kind of distraction, it is not seen as a target here.

In contrast to this situation, the detection technique for the driver's visual focus is rather well developed, and off-the-shelf products are readily available. These products are usually referred to as eye-trackers.

\subsection{Detection of visual distraction}

In order to detect visual distraction it is necessary to assess the driver's visual focus and to categorize if this visual focus contributes to a proper assessment of the road scene or not. Of course, there is a grey zone and more than two categories need to be used. Besides the visual focus itself, the time length for each visual focus also contributes to the distraction level.

Inferential detection of visual distraction is normally done by drawing conclusions from signals other than the desired state. This kind of technique is usually not effective for that kind of distraction, as mostly the driver's lane-keeping performance is assessed, which is then related to distraction. In terms of lane-keeping performance, there are many reasons for poor lane-keeping behaviour other than visual distraction, such as simply bad driving habits. Thus, there can be many causes of performance degradation, so that it is hard to establish a relation between visual distraction and performance, leading to many false positive decisions. Because of this, and owing to the properties of visual distraction and the latencies that are involved with performance assessment, inferential detection is not feasible.
This also suggests that a detection technique needs to be able to detect the visual focus of the driver instantaneously and needs to provide the distraction level with minimal latency. This, of course, places requirements on the sensor system used for visual focus detection.

It can be argued that the driver will still have the ability to maintain a given direction with the vehicle, which will keep the vehicle within the lane boundaries for a short time period ahead. That, of course, could relax the requirements on the detection scheme.

Testing with subjects has also shown that short glances by the driver at the road scene will have a positive effect on road scene attention. Similarly, glances at the periphery of the road scene have a negative affect, but only to a rather small extent. This suggests that eye glances do affect distraction, but, when occurring rarely, the effect can be neglected or can be dealt with as a detection uncertainty.

A driver monitoring camera can be used for the detection of visual distraction. It is often referred to as an eye-tracker. This eye-tracker provides the detection algorithm with information on head position, head pose, and eye gaze. Using these three values together with the fixed geometrical set-up of the vehicle, the driver's visual focus can be derived. The background of this technology is well described in reference [9].

\subsection{The detection scheme}

The detection algorithm consists of two parts (see Fig. 2). Firstly there is the distraction calculation (DC) which computes the instantaneous distraction level. Secondly there is the distraction decision-maker (DDM) which determines if the current distraction level represents a potentially distracted driver.

\subsubsection{Distraction calculation}

The distraction calculation (DC) utilizes the eye tracking data from the sensor, namely head position and head pose. Head position is a point in the vehicle coordinate system and the head pose is a unit vector that denotes the main direction of the driver's head. This direction can be compensated with the eye gaze information. Head position is denoted by $\boldsymbol{p}_{\mathrm{H}}$ and head pose is denoted by $\boldsymbol{e}_{\mathrm{G}}$. When the compensated head pose is used, $\boldsymbol{e}_{\mathrm{G}}$ will be denoted by $\boldsymbol{e}_{\mathrm{G}}^{\prime}$. Both $\boldsymbol{p}_{\mathrm{H}}$ and $\boldsymbol{e}_{\mathrm{G}}$ together define a ray that emerges from the forehead of the driver, which can be given by

$$
\boldsymbol{p}_{\text {ray }}\left(\varepsilon_{1}\right)=\boldsymbol{p}_{\mathrm{H}}+\boldsymbol{e}_{\mathrm{G}} \varepsilon_{1}
$$




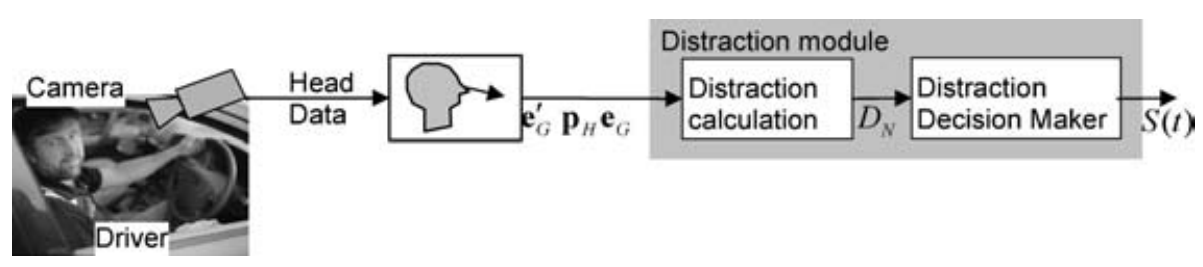

Fig. 2 Distraction detection scheme

where $\varepsilon_{1}$ is an arbitrary non-negative scalar value. Additionally, the head pose vector can be replaced by the compensated head pose vector.

In order to compute the visual focus of the driver, the geometrical structure of the vehicle needs to be available for the DC. It is assumed that the interior surfaces can be represented by a set of parallelograms as follows

$$
\mathscr{P}=\left\{P^{(1)}, P^{(2)}, \ldots, P^{(N)}\right\}
$$

where $P^{(i)}$ denotes the parallelograms and $N$ denotes the chosen number of parallelograms to represent the interior.

Each parallelogram $P^{(i)}$ can be defined by a position vector $\boldsymbol{p}_{\mathrm{par}}^{(i)}$ of one corner and the two vectors $\boldsymbol{\nu}_{1}^{(i)}$ and $\boldsymbol{v}_{2}^{(i)}$ that represent the non-parallel edges. All points within a parallelogram are given by

$$
\boldsymbol{p}_{\mathrm{par}, \mathrm{p}}^{(i)}\left(\lambda_{1}, \lambda_{2}\right)=\boldsymbol{p}_{\mathrm{par}}^{(i)}+\boldsymbol{v}_{1}^{(i)} \lambda_{1}+\boldsymbol{v}_{2}^{(i)} \lambda_{2}
$$

where $\lambda_{1}$ and $\lambda_{2}$ are scalar values between 0 and 1 .

Now, the visual focus can be derived by first finding the parallelograms within set $\mathscr{P}$ which intersects with $\boldsymbol{p}_{\text {ray }}$ and then computing the set $\mathscr{V}$ of intersection points. Clearly, there are as many intersection points as parallelograms that intersect.

In order to determine if an intersection point within a parallelogram exists, the following equation can be solved

$$
\left[\begin{array}{lll}
-\boldsymbol{e}_{\mathrm{G}} & \boldsymbol{v}_{1}^{(i)} & \boldsymbol{v}_{2}^{(i)}
\end{array}\right]^{-1}\left(\boldsymbol{p}_{\mathrm{H}}-\boldsymbol{p}_{\mathrm{par}}^{(i)}\right)=\left[\begin{array}{c}
\varepsilon_{1} \\
\lambda_{1} \\
\lambda_{2}
\end{array}\right]
$$

If a solution of equation (4) exists and the resulting values for both $\lambda_{1}$ and $\lambda_{2}$ are between 0 and 1 , there will be an intersection with $P^{(i)}$. Evaluating equation (4) for all $N$ parallelograms in $\mathscr{P}$, all intersecting parallelograms can be found.

The intersection points are then derived by evaluating (1) for every intersecting $P^{(j)}$ and are denoted by $\boldsymbol{p}_{\mathrm{I}}^{(j)}$, and accordingly

$$
\mathscr{V}=\left\{\boldsymbol{p}_{\mathrm{I}}^{(1)}, \ldots, \boldsymbol{p}_{\mathrm{I}}^{(M)}\right\}
$$

where $M$ is the number of intersecting $P^{(j)}$. The judgement could be made that the $\boldsymbol{p}_{\mathrm{I}}^{(j)}$ with the smallest non-negative $\varepsilon_{1}$ should be the driver's visual focus $\boldsymbol{p}_{\mathrm{VF}}$, which would be the computationally most feasible approach. Still, there is no evidence to support the hypothesis that the driver is really focusing on this point, given the uncertainty of the detection of the head pose $\boldsymbol{e}_{\mathrm{G}}$. Instead, none of the intersection points will be rejected or preferred on the basis of the distance from the driver.

It can be assumed that each $P^{(j)}$ contributes differently to the driver's distraction level. This is due to the fact that certain regions, although not part of the road scene ahead, are crucial for vehicle control, such as the instrument cluster or rear view mirror. Therefore, it is necessary to assign weighting factors to each $P^{(j)}$. To this end, scalar weights $w^{(j)}$ can be defined that map each $P^{(j)}$ into a scalar value between 0 and 1 , where 0 denotes no contribution and 1 means a large contribution. The weights are assigned in cooperation between human factors and test engineers, and are derived from test subject evaluations. Each member in the set $\mathscr{V}$ can now be augmented with the weighting information. The modified $\mathscr{V}^{\prime}$ is then given as

$$
\mathscr{V}^{\prime}=\left\{\left\{\boldsymbol{p}_{\mathrm{I}}^{(1)}, w^{(1)}\right\}, \ldots,\left\{\boldsymbol{p}_{\mathrm{I}}^{(M)}, w^{(M)}\right\}\right\}
$$

Owing to the uncertainties in the detection of $\boldsymbol{e}_{\mathrm{G}}$, the visual focus should be determined in favour of the driver, which means that the intersection parallelogram with the smallest $w^{(j)}$ was chosen. The visual focus of the driver, $\boldsymbol{p}_{\mathrm{VF}}$, that yields the least contribution to the driver distraction level is given by

$$
\boldsymbol{p}_{\mathrm{VF}}=\boldsymbol{p}_{\mathrm{I}}^{(k)}, \quad \text { with } k=\operatorname{index}\left[\min _{1 \leqslant j \leqslant M}\left(w^{(j)}\right)\right]
$$

Now that the visual focus and the weight are available, the normalized distraction level can be computed by generating the moving average over the weights for the visual focus point over time and yields

$$
D_{\mathrm{N}}(t)=\frac{1}{\mathrm{TH}} \int_{t-\mathrm{TH}}^{t} \min _{1 \leqslant j \leqslant M}\left(w^{(j)}\right) \mathrm{d} t
$$

Although this general equation gives a good approximation of the distraction level, there are several tuning parameters that need to be adjusted. First of 
all, there is the weighing function and then there is the time horizon TH. These values need to be found through testing and have to be investigated if there are driver-independent weighting factors.

Additionally, the equations cannot be used in their given continuous form. The integral needs to be time discretized and the weighing function needs to be meshed over a grid in order to obtain a representative dataset with discrete values. Tuning has also revealed that there are only a small number of regions in the weighing function that have different values associated with them. These regions are represented by the road scene, the road periphery, the mirrors, the instrumentation cluster, and the centre stack. All other regions could be binned to a weighing value of one. It is important to note that tuning of the weighing function to the correct assessment of the distraction level is essential.

\subsubsection{Distraction decision-maker}

The DDM uses the computed distraction level $D_{\mathrm{N}}$ in order to decide on the driver's actual distraction state. Since this is a pure binary decision, a continuous level has to be mapped into the distracted or not distracted state. This can be achieved by different techniques. The most simplistic approach is the choice of a threshold and yields the following decision function

$$
S(t)= \begin{cases}1, & D_{\mathrm{N}}(t)>\tau \\ 0, & D_{\mathrm{N}}(t) \leqslant \tau\end{cases}
$$

where $S(t)$ is the resulting driver state and $\tau$ is the decision threshold. While the decision threshold will work well for entering the distraction state, it will lead to a prolonged holding of that state, when the driver is actually turning the attention back to the road scene owing to the moving average based distraction level function. Since this is not desired behaviour, this simplistic approach could yield false intervention for a system that relies on low false positive rates.

Therefore, a dynamic or adaptive threshold should be used instead of the static threshold that was suggested above. Analysis of Fig. 1 suggests that a return from the distracted state should occur at rather high distraction levels if the distraction level function has decreased for a short while. Similarly, the distraction state should be entered at low distraction levels when the distraction level function is rising. An adaptive threshold that follows this scheme can be given as

$$
\tau(t)= \begin{cases}\tau_{\mathrm{H}} & \text { if } \int_{t-T_{\text {desc }}}^{t}-\operatorname{sign}\left(\frac{\mathrm{d} D_{\mathrm{N}}(t)}{\mathrm{d} t}\right) \mathrm{d} t \geqslant T_{\text {desc }} \\ \tau_{\mathrm{L}} & \text { otherwise }\end{cases}
$$

where $\tau_{\mathrm{H}}$ and $\tau_{\mathrm{L}}$ are the chosen thresholds for leaving and entering the distracted state respectively. The time $T_{\text {desc }}$ denotes the minimum time for a monotone descent of the distraction level. The modified decision-making function is then given by

$$
S(t)=\left\{\begin{array}{ll}
1, & D_{\mathrm{N}}(t)>\tau(t) \\
0, & D_{\mathrm{N}}(t) \leqslant \tau(t)
\end{array} \mid\right.
$$

The DDM will then output the estimated driver state.

\subsubsection{Lane-keeping module}

Lane-keeping assistance systems have been focused upon in recent years, leading to an abundance of different system layouts in terms of sensors, actuation, and HMI. According to Pilutti [10], it is useful to distinguish between warning, intervention, and control functions. Warnings do not directly alter the vehicle trajectory and require the driver to choose to act on the warning in order for the warning to have any effect. Intervention and control have the ability directly to affect the vehicle direction. Intervention has limited authority and is meant to augment driver commands, not replace them. Control is defined here as automatic control having full authority to steer the vehicle, which effectively removes the driver from the loop. For the development of the system described here it was found to be of utmost importance to make sure that the driver is in the loop and thereby responsible for vehicle control. The system described here is therefore classified as an intervention system, given the definition above.

The definition gives rise to the question of the appropriate actuator for lane keeping. There are in principle two ways to intervene with the vehicle in terms of steering, namely to provide a torque signal in the steering wheel and to provide a differential wheel angle (which could be accomplished on the front or rear axle). Even differential braking on the front or rear axle could be used in order to steer the vehicle. However, the driver perception of this approach is probably different to that of steering wheel torque intervention and must therefore be carefully investigated.

The additional torque command on the steering wheel can be accomplished via an additional electric 
motor acting on the steering shaft (see reference [11]) or via an electric power assisted steering gear (EPAS), as in the present paper.

The size of the angle or torque determines whether such a system is classed as an intervention system or a control system (i.e. whether it is possible for the driver to override the system). According to this definition, the system presented here falls into the category of intervention systems, since the driver perceives a torque feeling in the steering wheel which helps return the vehicle to the correct lane position but still can be overridden by the driver.

This is particularly important considering the sensor system employed. A single vision sensor monitoring the road scene can have difficulties in correctly identifying lane markers, which ultimately leads to false positive interventions. Therefore, it must be guaranteed that the driver can override the steering intervention.

Like the distraction module which consists of two separate parts, the lane-keeping assistance module consists of two modules (see Fig. 3). The lane position control module (LPC) calculates the target steering wheel angle and torque to keep the vehicle in the lane, while the lane departure module (LD) decides whether a lane departure is occurring or not.

\subsection{Lane position control}

The lane position control (LPL) module calculates the required torque offset in order to avoid a lane departure. This calculation is done in two separate steps. In step 1 the required steering wheel angle is calculated, and in step 2 this angle is compared with the actual steering wheel angle. The error between actual and optimal angle is then translated into an offset torque. This torque is called the offset torque, since it ultimately is superimposed on the current steering wheel angle torque produced by the EPAS steering gear.
The optimal steering angle $\delta_{\text {opt }}$ is calculated using curvature, heading angle, and vehicle lateral displacement at the front axle location

$$
\begin{aligned}
\delta_{\text {opt }}= & {\left[\left(\frac{b}{C_{\mathrm{f}}}-\frac{a}{C_{\mathrm{r}}}\right) \frac{m}{a+b} v^{2}+(a+b)\right] \beta \kappa } \\
& +K_{\theta \Delta}(v) \theta_{\Delta}+K_{y_{0}}(v) y_{0}
\end{aligned}
$$

The curvature can be seen as an outer disturbance, thus being feedforward, while heading angle and lateral displacement are feedback signals. The gains for the latter are linearly dependent on the vehicle speed, and the curvature gain is obtained from the steady state cornering equation. The controller scheme in equations (12) and (13) is only valid for stationary vehicle dynamics. This is, however, not a problem as the system is intended to intervene only when the driver is expected to be distracted, which is unlikely to occur during transient driving conditions. The torque calculation module consists of a feedforward and feedback path, with two vehicle speed dependent coefficients

$$
T_{\text {offset }}=K_{\mathrm{ff}}(v) \delta_{\mathrm{opt}}+K(v)\left(\delta_{\mathrm{opt}}-\delta\right)
$$

By splitting up the controller into optimal steering angle calculation and offset torque calculation, the tuning problem is divided into tuning of the steering wheel angle controller (step 1) and tuning of the actual lane position control (step 2).

Step 1 can ideally be done during low-speed manoeuvres on a test track. Here, a realistic target steering wheel angle is provided to the controller, and the controller gains as well as the torque saturation are tuned. (Naturally it is advantageous to have prior knowledge from a linear model about the stability margins of this control loop.) After step 1 has been completed, the lane position controller can be tuned on a test track. Here, the feedback gains for lane position and lateral velocity and the feedforward gain for curvature have to be tuned. Since lateral

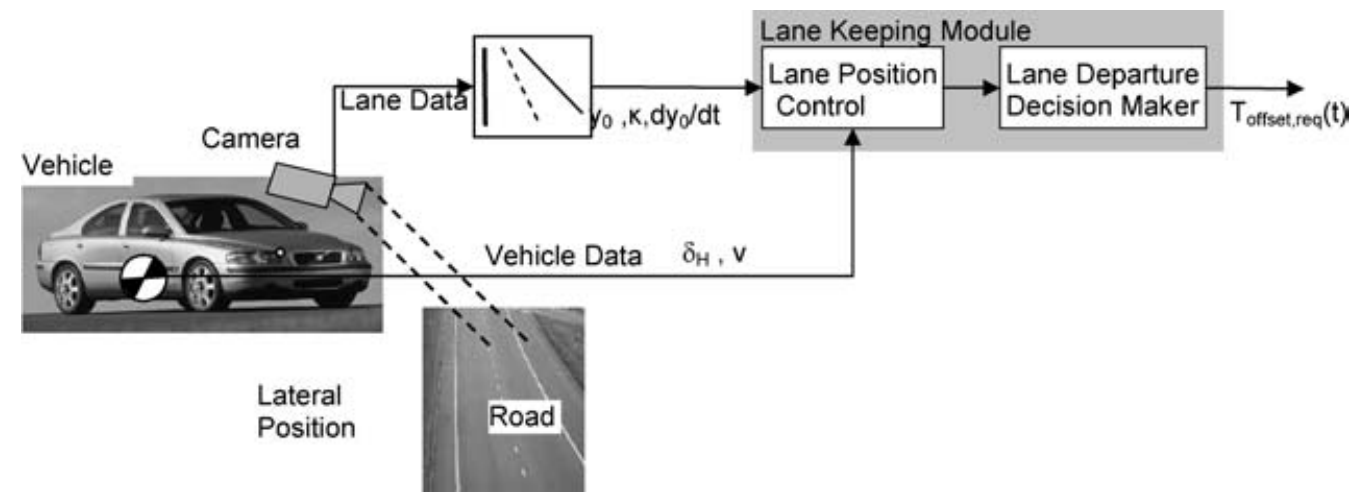

Fig. 3 Lateral vehicle control module 
vehicle dynamics is affected by vehicle velocity, these controller gains are vehicle speed dependent. This has not been the case for the steering wheel angle controller.

Splitting the controller into two separate parts has another advantage besides the tuning, which becomes obvious considering failsafe and system monitoring. As the main sensor for the lane position controller is a vision sensor which under adverse lighting conditions can confuse environmental features with lane markings, it is very convenient to be able to bound the optimal steering wheel angle contribution from the feedforward part of the curvature between the upper and lower thresholds. These thresholds can be made vehicle speed dependent.

Control methods such as linear quadratic Gaussian (LQG) control (linear quadratic Gaussian control synthesis, such as applied in reference [11]) or internal model control (IMC) constitute a model-based approach of the control problem and translate the actual tuning of feedback gain parameters to tuning of weighting matrices for LQG or system bandwidth for IMC. The tuning is therefore done in an offline manner and in-vehicle tuning becomes impractical. Ideally, there is no in-vehicle tuning required, which means that the (linear) vehicle model represents the vehicle dynamics accurately enough under all driving scenarios within the systems envelope. However, this cannot be expected.

\subsubsection{Lane departure decision-maker}

In the lane departure module, the decision is made whether a steering torque intervention from a lane departure point of view should take place or be aborted. The decision for starting an intervention is based upon when in time a lane departure will occur. Assuming constant lateral velocity, the time-to-lanecrossing (TTLC) prediction simply becomes

$$
T_{\text {TTLC }}=\operatorname{abs}\left(\frac{y_{0}}{\dot{y}_{0}}\right)
$$

When this time is below a predefined threshold (in this study a TTLC threshold of $0.4 \mathrm{~s}$ has been chosen), a lane crossing event is said to be detected.

Whether the intervention should be stopped is decided by whether the lateral position error to the lane centre, $y_{0}$, which refers to the lane that was occupied by the subject vehicle prior to the lane departure, and the heading error (vehicle angle towards lane marking), $\theta_{\Delta}$, are below a set of predefined thresholds. In other words, the intervention will stop when the vehicle has regained a stable lane position with low lateral velocity. It is worth mentioning that rather low values of these thresholds will result in longer interventions. Furthermore, it can be difficult for the system to obtain the same performance on curved roads as on straight roads. It is therefore advisable to include a timeout condition for the intervention.

\section{SYSTEM LAYOUT}

Figure 4 shows the basic layout of the entire system with the previously discussed function components. Both the distraction and lane-keeping modules report to an intervention module which principally constitutes an AND gate: if the vehicle is departing from the lane AND the driver is detected as distracted,

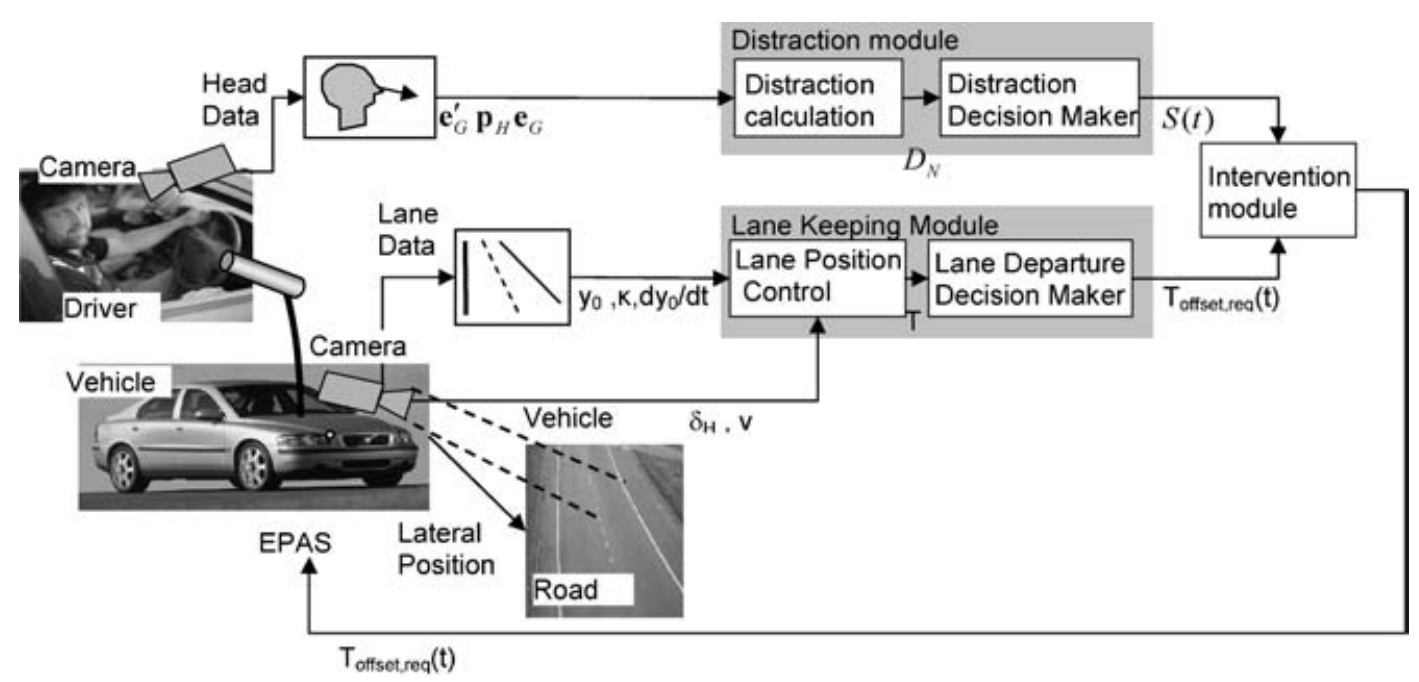

Fig. 4 System layout 
THEN an intervention is triggered by supplying the additional offset torque on the steering wheel.

The additional torque on the steering shaft can be produced in a number of different ways; here, an EPAS gear has been used. Such a steering gear is beneficial from a number of different points of view, among others low energy consumption and vehicle installation aspects, and as additional benefit it supports various steering-related active safety functions. Once vehicles are equipped with EPAS gears, additional required system components are two vision sensors, one for the driver and one for the road scene ahead of the vehicle.

\section{RESULTS AND DISCUSSION}

A decisive question in terms of customer acceptance and whether a lane-keeping system truly reduces the amount of single-vehicle accidents is whether the driver intuitively understands the system's function, without knowing that a lane-keeping system is active. Fourteen test participants were therefore asked to drive the test vehicle on a test track. The purpose of this small test population size was to prove the feasibility of the concept and to motivate larger test populations that will enable the quantification of the system performance in terms of true positive, false positive, and false negative interventions.

Prior to the test, the participants were not informed about the real intention of the experiment; rather, they were told that their driving capability during a set of distraction tasks was to be measured. The experiment was conducted on a closed track with no oncoming traffic. It is important to mention that the drivers claimed to have felt safe during the test, in spite of larger lane deviations which could lead to severe accidents in real traffic situations. This fact is important as the perception of danger and the judgement of the usefulness of a system are often connected. For a test participant it is hard to imagine how, for instance, a true positive intervention in a real traffic situation would be experienced.

In order to produce lane deviation, the test participants were severely distracted by several tasks. These tasks included changing radio channels, storing new channels in the radio memory, answering a cell phone, and finally sending text messages from a cell phone, which has been reported to cause significant distraction [12]. The system was judged in terms of true positive, false positive, false negative and true negative intervention.

\subsection{True positive performance}

One result of these experiments is shown in Fig. 5 where a series of pictures of a test participant is shown, together with the lane position of the vehicle, the intervention flags for both driver distraction and torque intervention, as well as the requested steering wheel offset torque and the resulting steering wheel angle. The grey shaded stripes indicate the instance in time when the picture was taken. The test subject was asked to start sending a text message from the mobile phone at $t=3 \mathrm{~s}$, which increases the driver's distraction level. The head vector points to a direction that has not been identified as a region of interest, i.e. is not pointing towards the road scene ahead. The increasing distraction level finally exceeds the predefined level at $t=4 \mathrm{~s}$ and the driver is judged to be distracted. However, the driver still keeps an appropriate lane position, which does not trigger a lane departure event until $2.5 \mathrm{~s}$ later at $t=6.5 \mathrm{~s}$. At $t=6.5 \mathrm{~s}$ both a driver distracted condition and lane departure are detected, leading to an intervention with a torque amplitude of nearly $3 \mathrm{~N} \mathrm{~m}$.

It should be noted that the maximum allowable torque has been restricted to $3 \mathrm{~N} \mathrm{~m}$ in order to allow the driver to override the system (this system is in accordance with the previously given definition classified as intervention and not as a control system). The intervention is active for approximately $2.5 \mathrm{~s}$ and stops when the vehicle heading angle and lane position error have settled below reasonable thresholds, as can be seen from the upper plot in Fig. 5.

\subsection{False positive performance}

One purpose of combining a distraction system with a lane-keeping support system has been to reduce the amount of false positive interventions. Such interventions can occur when both a lane departure is detected and the driver is detected as distracted at the same time. Figure 6 shows a false positive situation during the test where the driver did watch the road scene but the face vector pointed elsewhere over a sufficient amount of time to produce distraction detection. An intervention occurred owing to the driver's inability to keep a proper lane position, and thus the intervention was judged as being a true positive intervention.

\subsection{False negative performance}

The driver distraction portion of the system is the most delicate part and strongly relies on proper detection of the driver's face. This detection is made 


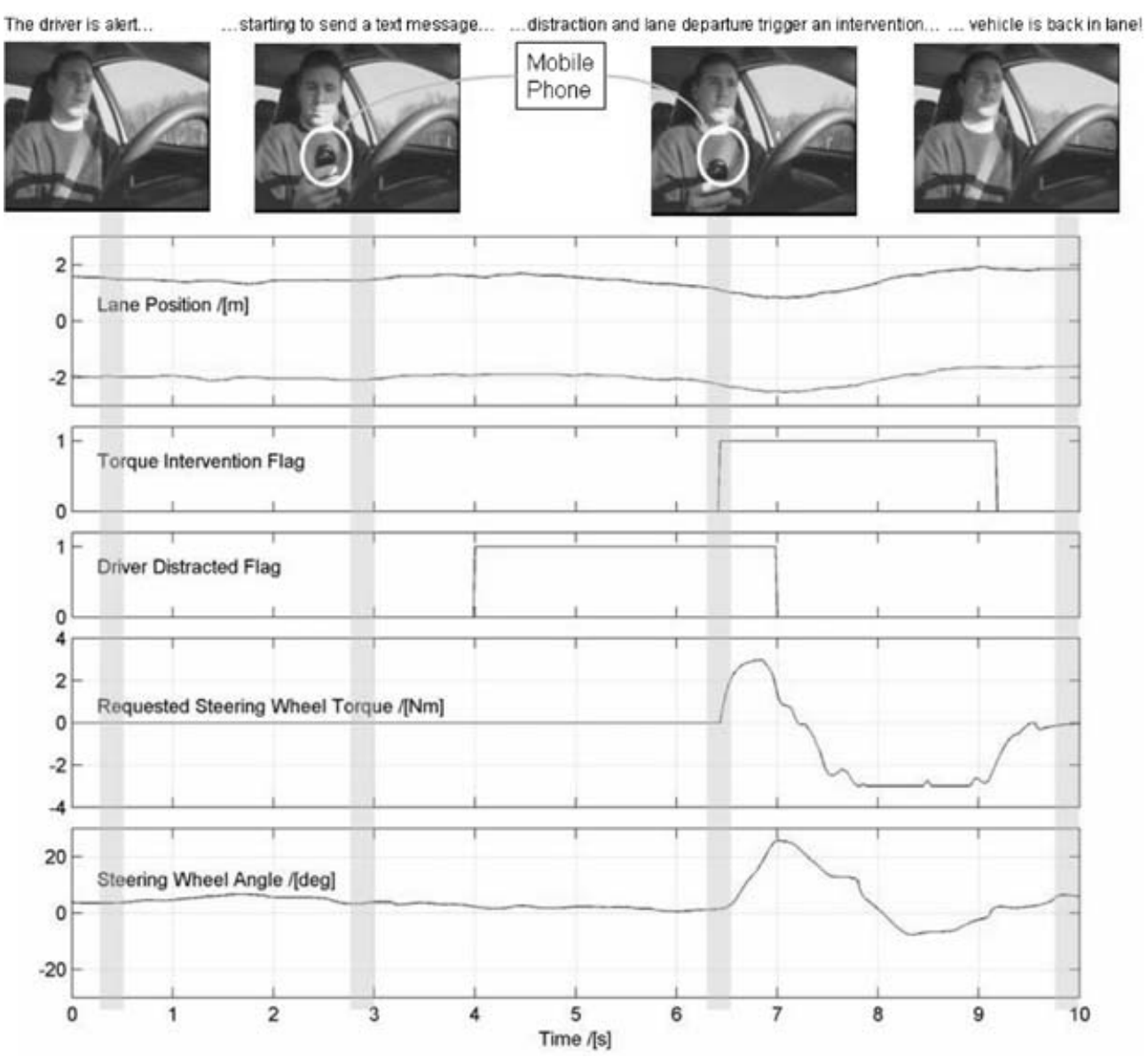

Fig. 5 Sequence of events during distraction-related lane departure (the grey stripes indicate the instance in time when the picture was taken)

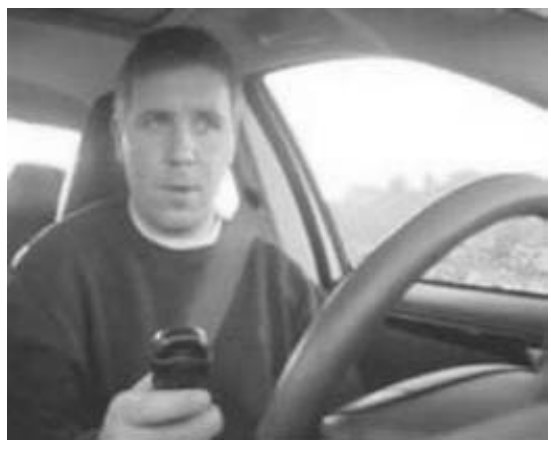

Fig. 6 'False positive' detection of distraction. The driver watches the road, although the face vector points elsewhere.

more difficult in inhomogeneous light conditions owing to, for instance, low sun or road lamps producing shadows on the driver's face. However, these disturbing factors are likely to produce false negative interventions only. No false positive intervention due to severs lighting conditions has been recorded. The false negative performance of the system still requires extensive improvement, as a large number of interventions have been missed owing to robustness and tracking envelope reasons.

\subsection{True negative performance}

There principally exist three separate types of true negative performance.

1. The driver watches the road scene and stays in lane.

2. The driver watches the road scene and leaves the lane.

3. The driver does not watch the road scene but stays in lane.

During testing of these three scenarios on the track, no occurrence other than just true negative has been reported. The reason for this behaviour lies in the functioning of the lane tracking system as well as the driver monitor. Both these systems are tuned to produce false negative rather than false positive interventions (i.e. not tracking is better than reporting the wrong thing). 


\section{CONCLUSIONS AND OUTLOOK}

A driver-distraction-based lane-keeping system has been presented, where emphasis has been put on the definition of visual distraction as well as the detection of the same through a sensor system. The sensor used is a vision sensor detecting both the driver's eye and gaze vectors. This system has been integrated with a lane-keeping assistance system with the aim of producing a lane-keeping system that only intervenes when a potentially dangerous lane departure event is detected, i.e. the driver is visually distracted and not attending to the road scene ahead while a lane departure is in progress. Thereby, the number of false interventions is reduced and at the same time it is possible to increase the authority of the intervention. This can be done as the intervention is based on a threat measure (driver inattention). This system has been tested on a test track, where 14 test participants have been deliberately distracted in order to produce a lane departure.

As a general result it can be said that it seems sufficient to base the distraction assessment on the driver's face direction, rather than taking gaze into account. Still, gaze direction is crucial for the detection of cognitive distraction ('see but not see'), but requires a much higher performance of the eye tracking device.

The system's performance in terms of false positive interventions is encouraging, as no such intervention has been detected during the tests. However, the number of false negative (missed) interventions was quite high owing to limitations in head tracking on account of light and tracking envelope restrictions.

Clearly, two steps now need to be taken.

1. The robustness of the eye and head tracking device to adverse light conditions has to be improved.

2. Tests with a larger test population that fits Volvo Cars customer profile need to be performed in order to quantify the system performance, as the feasibility of the concept has already been proven.

\section{REFERENCES}

1 Verunglückte nach Unfallart, ADAC http:// www.adac.de, 2000.

2 National survey of distracted and drowsy driving attitudes and behavior: 2002, National Highway Traffic Safety Administration (NHTSA) report 809566, 2003.

3 Green, P. Driver distraction, telematics design, and workload managers: safety issues and solutions, convergence. Paper 2004-21-0022, 2004.
4 Petterson, L., Fletcher, L., Barnes, N., and Zelinsky, A. An interactive driver assistance system monitoring the scene in and out of the vehicle. In Proceedings of the 2004 IEEE International Conference on Robotics and automation, New Orleans, Louisiana, 2004.

5 Lam, L. T. Distractions and the risk of car crash injury: the effect of drivers' age. J. Saf. Res., 2002, 33, 411-419.

6 Hancock, P. A., Lesch, M., and Simmons, L. The distraction effects of phone use during a crucial driving maneuver. Accident Analysis and Prev., 2003, 35, 501-514.

7 Patten, C. J. D., Kircher, A., Joakim Östlund, J., and Nilsson, L. Using mobile telephones: cognitive workload and attention resource allocation. Accident Analysis and Prev., 2004, 36, 341-350.

8 Johnson, M. B., Voas, R. B., Lacey, J. H., McKnight, A. S., and Lange, J. E. Living dangerously: driver distraction at high speed. Traff. Injury Prev., 2004, 5, 1-7.

9 Shinji Mita et al. Detection of the gaze direction of a driver using the time-varying image processing. JSAE Rev., 2003, 24, 347-353.

10 Pilutti, T. E. Lateral vehicle co-pilot to avoid unintended roadway departure. $\mathrm{PhD}$ Thesis, Department of Mechanical Engineering, The University of Michigan, 1997.

11 Naab, K. and Reichart, G. Driver assistance systems for lateral and longitudinal vehicle guidance heading control and active cruise support. Paper 78, AVEC 94, 2004.

12 deWaard, D., Hernandez-Gress, N., and Brockhuis, K. A. The feasibility of detecting phone-use related driver distraction. Int. J. Veh. Des., 2001, 26(1).

\section{APPENDIX}

\section{Notation}

$a, b \quad$ centre of gravity position (m)

$C_{\mathrm{f}}, C_{\mathrm{r}} \quad$ front and rear cornering stiffness (N/rad)

$D_{\mathrm{N}} \quad$ normalized distraction level

$\boldsymbol{e}_{\mathrm{G}} \quad$ unit gaze vector

$\boldsymbol{e}_{\mathrm{G}}^{\prime} \quad$ compensated unit gaze vector

$K \quad$ various gains

$\boldsymbol{p}_{\mathrm{H}} \quad$ head position

$\boldsymbol{p}_{\mathrm{I}} \quad$ intersection point

$\boldsymbol{p}_{\text {ray }} \quad$ line of sight

$\boldsymbol{p}_{\mathrm{VF}} \quad$ visual focus point

$p^{(i)} \quad i$ th parallelogram representing a region of the interior

$\begin{array}{ll}\mathscr{P} & \text { set of parallelograms } \\ S & \text { decision function }\end{array}$

$\mathrm{TH}$ time horizon (s)

$T_{\text {desc }} \quad$ minimum descent time (s)

$T_{\text {offset }} \quad$ offset torque $(\mathrm{N} \mathrm{m})$ 


$\begin{array}{llll}T_{\text {TTLC }} & \text { time to lane crossing (s) } & \beta & \text { gearing ratio } \\ v & \text { vehicle speed }(\mathrm{m} / \mathrm{s}) & \delta, \delta_{\text {opt }} & \text { steering wheel angles (rad) } \\ \boldsymbol{v}_{j}^{(i)} & \text { jth edge vector of the ith parallelogram } & \theta_{\Delta} & \text { heading error (rad) } \\ \mathscr{V}^{\prime} & \text { set of visual focus points } & \kappa & \text { road curvature }\left(\mathrm{m}^{-1}\right) \\ \mathscr{V}^{\prime} & \text { augmented set of visual focus points } & \tau & \text { decision threshold }\end{array}$

